\title{
Nasal Carriage Rate of Methicillin Resistant Staphylococcus aureus among Health Care Workers at a Tertiary Care Hospital in Kathmandu, Nepal
}

Sabita Khatri, ${ }^{1}$ Narayan Dutt Pant, ${ }^{2}$ Raju Bhandari, ${ }^{1}$ Krishma Laxmi Shrestha, ${ }^{1}$ Chandrika D Shrestha, ${ }^{3}$ Nabaraj Adhikari, ${ }^{1}$ Asia Poudel ${ }^{1}$

${ }^{1}$ Department of Microbiology, Goldengate International College, Kathmandu, Nepal, '2Department of Microbiology, Grande International Hospital, Kathmandu, Nepal, ${ }^{3}$ Kathmandu Medical College and Teaching Hospital, Kathmandu, Nepal.

\section{ABSTRACT}

Background: Methicillin-resistant Staphylococcus aureus is one of the most common causes of nosocomial infections.
Due to its multidrug resistant nature; infections due to Methicillin-resistant Staphylococcus aureus are often very difficult
to treat. Colonized health care workers are the important sources of Methicillin-resistant Staphylococcus aureus. The
objectives of this study were to determine the nasal carriage rate of Methicillin-resistant Staphylococcus aureus among
health care workers at Kathmandu Medical College and Teaching Hospital, Nepal and to assess their antimicrobial
susceptibility patterns.
Methods: A cross sectional study was conducted among 252 health care workers from July to November 2013.
Mannitol salt agar was used to culture the nasal swabs. Antimicrobial susceptibility testing was performed by Kirby-
Bauer disc diffusion technique following Clinical and Laboratory Standards Institute guidelines. Methicillin-resistant
Staphylococcus aureus strains were confirmed by using cefoxitin disc and by determining the minimum inhibitory
concentration of oxacillin by agar dilution method.
Results: Of 252 healthcare workers, $46(18.3 \%)$ were positive for Staphylococcus aureus among which $19(41.3 \%)$ were
Methicillin-resistant Staphylococcus aureus carriers. Overall rate of nasal carriage of Methicillin-resistant Staphylococcus
aureus was $7.5 \%$ (19/252).The higher percentages of lab personnel were nasal carriers of S. aureus (31.6\%) and
Methicillin-resistant Staphylococcus aureus (10.5\%). The percentages of nasal carriage of S. aureus (35.7\%) and Methicillin-
resistant Staphylococcus aureus (14.3\%) were highest in the health care workers from post operative department. Higher
percentage of Methicillin-resistant Staphylococcus aureus were susceptible toward amikacin (100\%) and vancomycin
(100\%) followed by cotrimoxazole (84.2\%).
Conclusions: High rates of nasal carriage of S. aureus and Methicillin-resistant Staphylococcus aureus were observed
among the healthcare workers, which indicate the need of strict infection control measures to be followed to control
the nosocomial infections.
Keywords: Health care workers; MRSA; nasal carriage; Nepal; Staphylococcus aureus.

\section{INTRODUCTION}

Methicillin-resistant Staphylococcus aureus (MRSA) has emerged as a burning problem and is a major cause of hospital acquired infections contributing to significantly high morbidity and mortality along with increased healthcare cost. ${ }^{1-3}$ However, MRSA is not only the problem in hospital but also a serious problem for community due to emergence of the community acquired MRSA. ${ }^{4}$ Health care workers(HCWs) colonized with MRSA may carry these virulent hospital strains in their nose and skin and may transmit these organisms to the community creating a more dreadful situation. ${ }^{3,5}$ Thus there is significant role of nasal MRSA carriers in transmission of the pathogen and the health care workers may serve in cross transmission of the community acquired MRSA and hospital acquired MRSA. ${ }^{4}$ It is important to study the nasal carriage rate of MRSA among healthcare workers. In this study we determined the nasal carriage rates of S. aureus and MRSA among health care workers. 


\section{METHODS}

A cross sectional study was conducted among 252 HCWs (doctor, nurse, attendant, health assistant, lab personnel) from July to November 2013 at Kathmandu Medical College and Teaching Hospital, Kathmandu, Nepal. Single nasal swab was collected from each health worker. Sterile cotton swabs soaked in sterile normal saline were used to collect the samples from anterior nares. The swabs obtained were inoculated into mannitol salt agar and were incubated at $37^{\circ} \mathrm{C}$ for 24 hrs. The mannitol fermenting colonies (yellow colored) which were gram-positive cocci, catalase positive and coagulase positive were identified as Staphylococcus aureus. For screening of MRSA cefoxitin disc $(30 \mu \mathrm{g})$ was used. The strains showing resistance to cefoxitin disc were reported to be MRSA. ${ }^{6}$ Further, confirmation of MRSA was done by determining minimum inhibitory concentration (MIC) of oxacillin by agar dilution method.

All the MRSA isolates detected with the help of cefoxitin disc were subjected to invitro determination of minimum inhibitory concentration (MIC) of oxacillin by agar dilution method following the techniques suggested by Andrews. ${ }^{7}$ The results were interpreted according to Clinical and Laboratory Standards Institute (CLSI) guidelines. ${ }^{6}$ The different dilutions of oxacillin used were 0.125 to $128 \mu \mathrm{g} / \mathrm{ml}$.

Antimicrobial susceptibility testing of isolates was performed by Kirby-Bauer disc diffusion technique as suggested by CLSI guidelines. ${ }^{6}$ The antibiotic discs used were cefoxitin $(30 \mu \mathrm{g})$, amikacin $(30 \mu \mathrm{g})$, ciprofloxacin $(30 \mu \mathrm{g})$, azithromycin $(15 \mu \mathrm{g})$, co-trimoxazole $(25 \mu \mathrm{g})$, ceftriaxone $(30 \mu \mathrm{g})$, vancomycin $(30 \mu \mathrm{g})$, penicillin $\mathrm{G}(10$

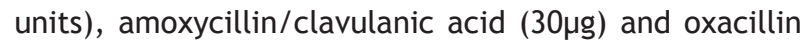
$(1 \mu \mathrm{g})$.

The data obtained were analyzed using SPSS version 19. Chi-square test was used.

Before commencing of the study the ethical approval was taken from the ethical committee of Kathmandu Medical College. Written consent was obtained from all the participants.

\section{RESULTS}

Of total 252 samples processed, S. aureus was isolated from 46 samples (18.3\%), among which, 19 (41.3\%) were MRSA. Overall rate of nasal carriage of MRSA was $7.5 \%$ $(19 / 252)$. The MICs of oxacillin for all the MRSA isolates detected with the help of cefoxitin disc ranged from $4 \mu \mathrm{g} / \mathrm{ml}$ to $128 \mu \mathrm{g} / \mathrm{ml}$.

Table 1. Age and sex wise distribution of nasal carriage rates of S. aureus and MRSA.

\begin{tabular}{|llll}
$\begin{array}{l}\text { Age groups } \\
\text { (years) }\end{array}$ & $\begin{array}{l}\text { Total num- } \\
\text { ber of sam- } \\
\text { ples }\end{array}$ & S. aureus & MRSA \\
\hline $15-25$ & 106 & $21(19.8 \%)$ & $10(9.4 \%)$ \\
\hline $26-35$ & 98 & $15(15.3 \%)$ & $4(4.1 \%)$ \\
$36-45$ & 27 & $9(33.3 \%)$ & $4(14.8 \%)$ \\
\hline Above 45 & 21 & $1(4.8 \%)$ & $1(4.8 \%)$ \\
\hline
\end{tabular}

\section{Table 2. Nasal carrier rate of MRSA and S. aureus among different subgroups of study population.}

\begin{tabular}{|c|c|c|c|c|c|}
\hline \multirow[t]{2}{*}{ Total samples } & \multirow[t]{2}{*}{ Occupation } & \multirow[t]{2}{*}{$\begin{array}{l}\text { Total number of } \\
\text { samples }\end{array}$} & \multicolumn{2}{|c|}{ Positive cases } & \multirow[b]{2}{*}{ Total S. aureus } \\
\hline & & & MSSA & MRSA & \\
\hline \multirow[b]{5}{*}{252} & Doctor & 63 & 9 & $4(6.4 \%)$ & $13(20.6 \%)$ \\
\hline & Nurse & 121 & 10 & $12(9.9 \%)$ & $22(18.2 \%)$ \\
\hline & Attendant & 42 & 4 & $1(2.4 \%)$ & $5(11.9 \%)$ \\
\hline & Health assistant & 7 & 0 & 0 & 0 \\
\hline & Lab personnel & 19 & 4 & $2(10.5 \%)$ & $6(31.6 \%)$ \\
\hline \multirow[t]{4}{*}{ Total samples } & \multirow[t]{2}{*}{ Departmen } & \multicolumn{2}{|c|}{ Total no. of participants } & MRSA & Total S. aureus \\
\hline & & & & $2(3.9 \%)$ & $10(19.2 \%)$ \\
\hline & ICU & 48 & & $3(6.3 \%)$ & $7(14.6 \%)$ \\
\hline & ER & 24 & & $3(12.5 \%)$ & $6(25 \%)$ \\
\hline 252 & OPD & 50 & & $5(10 \%)$ & $8(16 \%)$ \\
\hline
\end{tabular}


Nasal Carriage Rate of Methicillin Resistant Staphylococcus Aureus among Health Care Workers

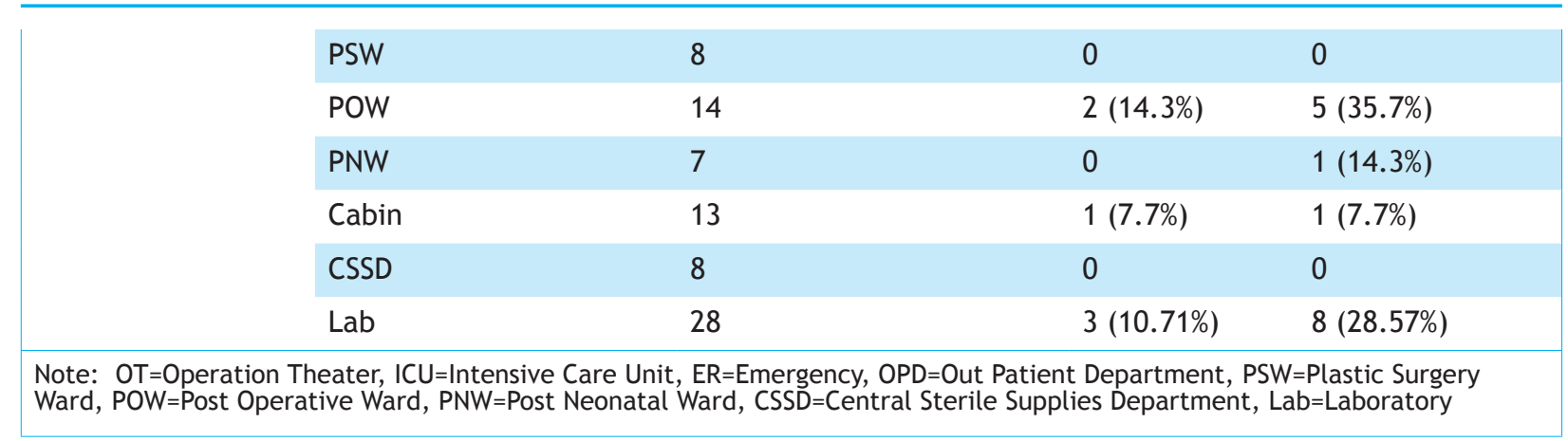

\section{Table 4. Antibiotic susceptibility patterns shown by S. aureus isolated.}

\begin{tabular}{|c|c|c|c|c|}
\hline \multirow[b]{3}{*}{ Antibiotics used } & \multicolumn{4}{|c|}{ Number of S. aureus isolates } \\
\hline & \multicolumn{2}{|c|}{$\operatorname{MRSA}(n=19)$} & \multicolumn{2}{|c|}{$\operatorname{MSSA}(n=27)$} \\
\hline & Sensitive & Resistant & Sensitive & Resistant \\
\hline Oxacillin & 0 & $19(100 \%)$ & $27(100 \%)$ & 0 \\
\hline Cefoxitin & 0 & $19(100 \%)$ & $27(100 \%)$ & 0 \\
\hline Amikacin & $19(100 \%)$ & 0 & $27(100 \%)$ & 0 \\
\hline $\begin{array}{l}\text { amoxycillin/clavulanic } \\
\text { acid }\end{array}$ & $2(10.5 \%)$ & $17(89.5 \%)$ & $6(22.2 \%)$ & $21(77.8 \%)$ \\
\hline Azithromycin & $5(26.3 \%)$ & $14(73.7 \%)$ & $7(25.9 \%)$ & $20(74.1 \%)$ \\
\hline Ceftriaxone & 0 & $19(100 \%)$ & $24(88.9 \%)$ & $3(11.1 \%)$ \\
\hline Ciprofloxacin & $13(68.4 \%)$ & $6(31.6 \%)$ & $21(77.8 \%)$ & $6(22.2 \%)$ \\
\hline Co-trimoxazole & $16(84.2 \%)$ & $3(15.8 \%)$ & $17(63.0 \%)$ & $10(37.0 \%)$ \\
\hline Penicillin & 0 & $19(100 \%)$ & 0 & $27(100 \%)$ \\
\hline Vancomycin & $19(100 \%)$ & 0 & $27(100 \%)$ & 0 \\
\hline \multicolumn{5}{|c|}{ 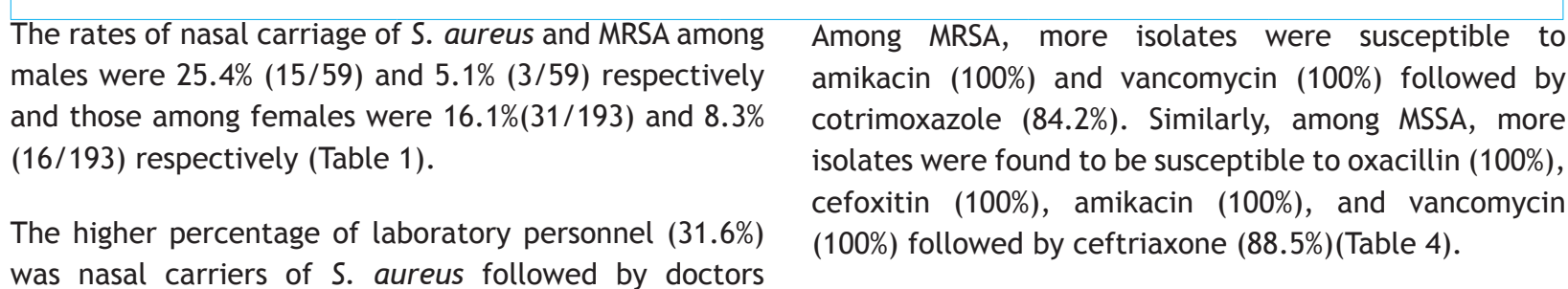 } \\
\hline
\end{tabular}
(20.6\%) and nurses $(18.2 \%)$. Similarly, the higher percentage of laboratory personnel $(10.5 \%)$ were nasal carriers of MRSA followed by nurses $(9.9 \%)$ and doctors (6.4\%) (Table 2).

The percentage of nasal carriage of $S$. aureus was higher among the HCWs from post operative ward (35.7\%), followed by those working in laboratory $(28.6 \%)$ and emergency $(25 \%)$. Similarly, the percentage of nasal carriage of MRSA was also higher among the HCWs from post operative ward $(14.3 \%)$, followed by emergency (12.5\%) and laboratory (10.7\%)(Table 3).

\section{DISCUSSION}

Staphylococcus aureus is one of the most common causes of serious infections in the community as well as the hospital. It is also a common normal flora of human beings, mainly presenting in the skin and anterior nares. Among healthcare workers, the average rate for carriage of S. aureus has been reported to be $23.7 \%$ and that of MRSA has been shown to be $4.6 \% .^{8}$ In our study, the nasal carriage rate of S. aureus among HCWs was found to be $18.3 \%$, which is in accordance with the results reported by Sah et al. (20.4\%), ${ }^{5}$ Khanal et al. (15.7\%), ${ }^{4}$ Akhtar $(18.2 \%)^{9}$ and Radhakrishna et al. (17.5\%). ${ }^{10}$ However, 
higher carriage rates of S. aureus were reported by Pant et al. (90\%), ${ }^{3}$ Pant and Rai (43.8\%), ${ }^{11}$ Shakya et al. (25\%), ${ }^{12}$ Shrestha et al. $(27.1 \%)^{13}$ and Farzana et al. (48\%). ${ }^{14}$ But very low rate in comparison to our study was reported by Planta et al. (7.7\%). ${ }^{15}$ This disparity in the carriage rates reported by different authors may be attributed to quality of the samples used, population under study, study location and techniques used for detection of nasal carriage. ${ }^{16-18}$

In our study, the overall rate of nasal carriage of MRSA was found to be $7.5 \%$, which was in accordance with the finding by Malini et al. (8\%). ${ }^{19}$ However, higher rates of MRSA carriage among HCWs were reported by Pant et al. ${ }^{3}$ (54\%), ${ }^{3}$ Shakya et al. $(10 \%)^{12}$ and Shibabaw et al. (12.7\%). ${ }^{20}$ While lower carriage rates of MRSA than our finding were observed in the studies performed by Radhakrishna et al.(2.5\%), ${ }^{10}$ Shrestha et al. $(2.3 \%)^{13}$ and Khanal et al. (3.4\%). ${ }^{4}$ Health care workers are the sources of MRSA, who are responsible for transferring of this pathogen to the patients, ${ }^{3}$ and the haphazard use of the antibiotics may have contributed to the emergence of the MRSA. Further, the local prevalence of MRSA may have attributed to the difference in carriage rates reported by different authors. ${ }^{4}$

On the basis of different professions among HCWs, the higher percentage of lab personnel (31.6\%) were nasal carriers of S. aureus followed by doctors (20.6\%) and nurses $(18.2 \%)$. Similarly, the higher percentage of lab personnel $(10.5 \%)$ were MRSA carriers followed by nurses (9.9\%) and doctors(6.4\%). But in a study by Khanal et al., higher percentage of doctors $(20.8 \%)$ were S. aureus carriers and higher percentage of nurses $(7.8 \%)$ were MRSA carriers. ${ }^{4}$ Similarly, Shibabaw et al. reported the MRSA carriage percentage to be highest among nurses (21.2\%), followed by doctors (12.5\%) and laboratory technicians $(12.5 \%)$ and $S$. aureus carriage percentage to be highest among doctors (37.5\%) followed by nurses (34.6\%) and laboratory technicians (25\%). ${ }^{20}$ The highest percentages of $S$. aureus and MRSA carriage found among laboratory personnel in our study might be due to the contact of the laboratory personnel to the infected samples received from different wards. Among HCWs, the occupation is the sufficient risk factor for nasal carriage of MRSA. ${ }^{17}$ The contamination of the hands due to contact with pathogens during laboratory works or due to direct contact with the patients or their surroundings are the main reasons for high rates of colonization of S. aureus and MRSA in HCWs. ${ }^{21}$ The high rate of nasal carriage of MRSA among HCWs indicates the high chances of transmission of these pathogens to the patients during patient-care. ${ }^{4}$ Further, the HCWs who are MRSA carriers can disseminate the pathogen to community and also possess the high risk of getting endogenous infections. ${ }^{3}$

In our study, the percentage of nasal carriage of S. aureus was highest in HCWs from post operative ward (35.7\%), followed by those from laboratory $(28.6 \%)$ and emergency (25\%). Similarly, the percentage of nasal carriage of MRSA was also highest in HCWs from post operative ward (14.28\%), followed by those from emergency (12.5\%) and laboratory $(10.71 \%)$. The poor sanitation of the different departments and the poor hygiene practice of the health care workers in different departments may be the reasons behind the higher prevalence of carriage rates in staffs from different departments. ${ }^{20}$ Since, in our study high rates of nasal carriage of $S$. aureus and MRSA were found in the staffs from post operative wards; the chances of surgical wound infections with these organisms (if strict hand hygiene is not followed) is high in our setting and may complicate the treatment. The nasal MRSA carriage among the staffs from emergency suggests the possible invasion of the community acquired methicillin resistant Staphylococcus aureus (CA-MRSA) into hospital environment. ${ }^{4}$ Due to multidrug resistance shown by MRSA, the treatment options for infections caused by this organism are limited. ${ }^{3}$ Antimicrobial susceptibility testing of the MRSA in our study indicated that the methicillin resistant strains were highly susceptible to vancomycin $(100 \%)$, amikacin $(100 \%)$ and cotrimoxazole (84.2\%) suggesting these drugs as the possible agents of empirical therapy for treatment of infections caused by MRSA in our settings.

\section{CONCLUSIONS}

High rate of nasal carriage of MRSA was observed among the healthcare workers. HCWs of postoperative wards had the highest carriage rate. There is need of infection control program targeting HCWs in this hospital.

\section{ACKNOWLEDGEMENTS}

The authors would like to thank, Kathmandu Medical College and Teaching Hospital, Kathmandu, Nepal for providing the platform to conduct this study. The authors would also like to thank all participants of this study and the laboratory staffs for their help during the study.

\section{REFERENCES}

1. Zafar A, Stone M, Ibrahim S, Parveen Z, Hasan Z, Khan E, et al. Prevalent genotypes of meticillin-resistant Staphylococcus aureus: report from Pakistan. J Med Microbiol. 2011;60(1):56-62. [PubMed] 
2. Moran GJ, Krishnadasan A, Gorwitz RJ, Fosheim GE, McDougal LK, Carey RB, et al. Methicillin-resistant S. aureus infections among patients in the emergency department. N Engl J Med. 2006;355(1):666-74. [PubMed]

3. Pant ND, Sharma M. Carriage of methicillin resistant Staphylococcus aureus and awareness of infection control among health care workers working in intensive care unit of a hospital in Nepal. Braz J Infect Dis. 2016;20(2):218-9.

4. Khanal R, Sah P, Lamichhane P, Lamsal A, Upadhaya S, Pahwa VK. Nasal carriage of methicillin resistant Staphylococcus aureus among health care workers at a tertiary care hospital in Western Nepal. Antimicrob Resist Infect Control. 2015;4:39.

5. Sah P, Rijal KR, Shakya B, Tiwari BR, Ghimire P. Nasal Carriage Rate of Staphylococcus aureus in Hospital Personnel of National Medical College and Teaching Hospital and their Antibiotic Susceptibility Pattern. JHAS. 2013;3(1):21-3.

6. Clinical Laboratory Standards Institute (CLSI). CLSI document M100S-S23. Performance standards for antimicrobial susceptibility testing: Twenty third informational supplement ed. Wayne: CLSI; 2013.

7. Andrews MJ. Determination of minimum inhibitory concentrations. J Antimicrob Chemother. 2001;48 (Suppl 1):5-16. [PubMed]

8. Albrich WC, Harbarth S. Health-care workers: source, vector, or victim of MRSA? Lancet Infect Dis. 2008;8:289301. [PubMed]

9. Akhtar N. Staphylococcal nasal carriage of health care workers. J Coll Physicians Surg Pak. 2010;20(7):439-43. [PubMed]

10. Radhakrishna M, D’Souza M, Kotegadde S, Saralaya KV, Kotian MS. Prevalence of methicillin resistant Staphylococcus aureus carriage amongst health care workers of critical care units in Kasturba Medical College Hospital, Mangalore, India. J Clin Diagn Res. 2013;7(12):2697-700. [PubMed]

11. Pant J, Rai SK. Occurrence of Staphylococcus aureus in hospital environment and staffs in teaching hospital in Kathmandu, Nepal. J NAMLS.2007;8(1):72-3.

12. Shakya B, Shrestha S, Mitra T. Nasal carriage rate of methicillin resistant Staphylococcus aureus among at Nepal Medical college Teaching hospital, Birgunj, Nepal. Nepal Med Coll J. 2010;12(1):26-9. [PubMed]

13. Shrestha B, Pokhrel BM, MohapatraTM. Staphylococcus aureus nasal carriage among health care workers in a Nepal
Hospital. Brazi J Infect Dis. 2009;13(5):322. [PubMed]

14. Farzana K, Rashid Z, Akhtar N, Sattar A, Khan JA, Nasir B. Nasal Carriage of Staphycocci in Health Care Workers: antimicrobial susceptibility profile. Pak J Pharm Sci. 2008;21(3): 290-4. [PubMed]

15. Planta PM, Laiño AGG, Alqueza NB, Gonzales L. Nasal Carriage of Staphylococcus aureus among Pediatric Health Care Workers in a Pediatric Intensive Care Unit. PIDSP Journal. 2012;13(1):44-50.

16. Adesida SA, Abioye OA, Bamiro BS, Brai BI, Smith SI, Amisu KO, et al. Associated risk factors and pulsed field gel electrophoresis of nasal isolates of Staphylococcus aureus from medical students in a tertiary hospital in Lagos, Nigeria. Braz J Infect Dis. 2007;11(1):63-9. [PubMed]

17. Askarian M, Zeinalzadeh A, Japoni A, Alborzi A, Memish ZA. Prevalence of nasal carriage of methicillin-resistant Staphylococcus aureus and its antibiotic susceptibility pattern in healthcare workers at Namazi Hospital, Shiraz, Iran. Int J Infect Dis. 2009; 11(1):e241-7. [PubMed]

18. Onanuga A, Temedie TC. Nasal carriage of multi-drug resistant Staphylococcus aureus in healthy inhabitants of Amassoma in Niger delta region of Nigeria. Afr Health Sci. 2011;11(2):176-81. [PubMed]

19. Malini J, Shruti AH, Padmavathy M, Umapathy BL, Navaneeth BV, Keerthi MJ, et al. Methicillin resistant Staphylococccus aureus carriage among health care workers in Tertiary health care hospital. J Clin Diagn Res. 2012;6(5):791- 3 .

20. Shibabaw A, Abebe T, Mihret A. Nasal carriage rate of methicillin resistant Staphylococcus aureus among Dessie Referral Hospital Health Care Workers; Dessie, Northeast Ethiopia. Antimicrob Resist Infect Control. 2013;2(1):25. [PubMed]

21. Saadatian-Elahi M, Tristan A, Laurent F, Rasigade JP, Bouchiat C, Ranc AG et al. Basic rules of hygiene protect health care and lab workers from nasal colonization by Staphylococcus aureus: an international cross-sectional study. PLoS One. 2013;8(12):e82851. [PubMed] 